\title{
Deterioro de la salud por causa de las vibraciones mecánicas en los centros de trabajo de la zona de Tecamachalco
}

\section{Health damage due to mechanical vibrations in the work centers of the Tecamachalco zone}

PÉREZ-VILLEGAS，Manuel†*，TÉLLEZ-HERNÁNDEZ，Rubén，SÁNCHEZ-BAÑUELOS，José Sebastián y TENORIO-CRUZ, Fermín

Universidad Tecnológica de Tecamachalco

ID $1^{\text {er }}$ Autor: Manuel, Pérez-Villegas / ORC ID: 0000-0002-4204-3813, Researcher ID Thomson: ABC-8100-2020, CVU CONACYT ID: 818732

ID $1{ }^{\text {er }}$ Coautor: Rubén, Téllez-Hernández / ORC ID: 0000-0002-6184-3226, Researcher ID Thomson: S-8747-2018, CVU CONACYT ID: 665940

ID $2^{\text {do }}$ Coautor: José Sebastián, Sánchez-Bañuelos / ORC ID: 0000-0002-3577-7384

ID $3^{\text {er }}$ Coautor: Fermín, Tenorio-Cruz / ORC ID: 0000-0002-8727-1502, Researcher ID Thomson: S-8629-2018, CVU CONACYT ID: 84030

DOI: $10.35429 / \mathrm{JOHS} .2020 .23 .7 .11 .17$

Recibido Octubre 10, 2020; Aceptado Diciembre 29, 2020

\section{Resumen}

El objetivo de esta investigación es reunir los elementos suficientes para implementar un programa de monitoreo y control de vibraciones en diversas empresas de la región de Tecamachalco, de acuerdo a los límites de exposición permisibles sugeridos por la norma ISO 2631-1 y en cumplimiento con la Norma Oficial Mexicana NOM-024STPS-2001. Se trata de asegurar el bienestar de los trabajadores, disminuyendo el riesgo laboral y los potenciales accidentes que pueden ocurrir en consecuencia. Se realizó una investigación para determinar las condiciones de operación de las empresas, después se realizó un análisis para determinar los niveles de vibración existentes y en base a ello, realizar la propuesta más adecuada para disminuir los riesgos de deterioro de la salud de los trabajadores por causa de las vibraciones de los equipos. La aportación de la presente investigación consiste en concientizar a los empresarios de los riesgos que tienen los trabajadores al estar en contacto con las vibraciones mecánicas por periodos prolongados de tiempo, con sus repercusiones a mediano y largo plazo.

Vibraciones, Exposición, Riesgo laboral

\begin{abstract}
The objective of this research is to gather sufficient elements to implement a vibration monitoring and control program in various companies in the Tecamachalco region, in accordance with the permissible exposure limits suggested by ISO 2631-1 and in compliance with the Official Mexican Norm NOM-024-STPS-2001. It is about ensuring the wellness of workers, reducing the occupational risk and potential accidents that may occur as consequence. An investigation was done to determine the operating conditions of the companies, then an analysis was done to determine the existing vibration levels and based on this, make the most appropriate proposal to reduce the risks of deterioration of the health of workers by cause of equipment vibrations. The contribution of the research consists of making employers aware of the risks that workers have when being in contact with mechanical vibrations for long periods of time, with their repercussions in the medium and long term.
\end{abstract}

Vibrations, Exposure, Occupational risk

Citación: PÉREZ-VILLEGAS, Manuel, TÉLLEZ-HERNÁNDEZ, Rubén, SÁNCHEZ-BAÑUELOS, José Sebastián y TENORIO-CRUZ, Fermín. Deterioro de la salud por causa de las vibraciones mecánicas en los centros de trabajo de la zona de Tecamachalco. Revista de Ciencias de la Salud. 2020. 7-23: 11-17

*Correspondencia al Autor (correo electrónico: mantenimiento-utt@ outlook.es)
$\dagger$ Investigador contribuyendo como primer Autor 


\section{Introducción}

Las vibraciones de origen mecánico representan un riesgo para la salud de las personas, cuando éstas se exponen al operar herramientas, equipos o máquinas que generan vibraciones. Desde una perspectiva de la Seguridad y Salud en el Trabajo, se ha demostrado que la exposición a vibraciones críticas en cuerpo entero, combinada con otros factores, tales como demandas posturales incómodas, pueden afectar sensiblemente la salud y el bienestar de los trabajadores. Con el fin de intervenir en este riesgo se han elaborado normas de carácter internacional que permiten la identificación, evaluación e intervención de los riesgos asociados a las vibraciones mecánicas. (Castro et al., 2016).

El objetivo de esta investigación es reunir los elementos suficientes para implementar un programa de monitoreo y control de vibraciones en diversas empresas de la región de Tecamachalco, debido a los altos niveles de vibración presentados, de acuerdo a los límites de exposición permisibles sugeridos por la norma ISO 2631-1 y en cumplimiento con la Norma Oficial Mexicana NOM-024-STPS2001. Se trata de asegurar el bienestar de los trabajadores, disminuyendo el riesgo laboral y los potenciales accidentes que pueden ocurrir en consecuencia.

Con la implementación de éste programa se busca disminuir el efecto que tienen las vibraciones mecánicas en el cuerpo del trabajador, que son transmitidas de las máquinas al cuerpo de los trabajadores y que lo afectan directamente, causando deterioro físico, disminuyendo su rendimiento laboral y propiciando condiciones inseguras de trabajo. Todo esto afecta directamente la competitividad de las empresas.

Para esta investigación se cuenta con el equipo analizador de vibraciones: ADASH A43000 - VA3, equipado con el acelerómetro $100 \mathrm{mV} / \mathrm{g}$ p/propósito general de la marca ADASH y personal certificado para el análisis.

\section{Vibraciones}

La palabra vibración se refiere a los movimientos oscilatorios (en todas las direcciones) de las estructuras, de los sistemas mecánicos o de sus componentes.
Las vibraciones son causadas siempre por fuerzas no continuas, esto es, fuerzas que puedan ser oscilatorias en magnitud o dirección, o fuerzas que se aplican o liberan de repente. En general, la vibración puede describirse como un movimiento oscilatorio de un sistema. El movimiento puede ser un movimiento armónico simple o puede ser extremadamente complejo. El sistema puede ser gaseoso, líquido o sólido. (Henao, 2007, p. 81).

De acuerdo con Falagán (2000), la vibración es un fenómeno físico no deseable, aunque en ocasiones se produce para hacer funcionar un dispositivo (martillos mecánicos, cintas transportadoras vibratorias, tamices vibradores, etc.) y en esos casos el ruido resultante es inevitable, debiendo procederse al aislamiento necesario (Jesús \& Ciro, 2016).

\section{Efectos de las vibraciones sobre el cuerpo humano}

En muchos estudios desarrollados en campo o laboratorios se han investigado las respuestas objetivas y subjetivas de los seres humanos a las vibraciones. Los objetivos de estos estudios han sido diversos, desde la identificación de los factores que afectan las respuestas biodinámicas en presencia de vibraciones (Nawayseh \& Griffin, 2012), hasta los factores que afectan el confort y la salud (Basri \& Griffin, 2012, 2013).

Así mismo investigaciones como las desarrolladas por Ramakrishnan, Milosavljevic \& Sullivan (2011), en las que se encuentra una relación lineal negativa entre la masa corporal y los niveles de exposición a vibraciones (Ramakrishnan, Milosavljevic, \& Sullivan, 2011 y Blood, Ploger, Yost, Ching \& Johnson, 2010).

Existen otras molestias que afectan a la salud humana tales como: irritabilidad, tensión nerviosa, lo cual origina fatiga precoz, puede causar molestia, accidentes y favorecer el ausentismo, disminución de la resistencia eléctrica de la piel, reducción de la actividad gástrica, aceleración temporal del ritmo respiratorio y cardíaco con aumento de la presión arterial, si es extremadamente excesivo, pueden producirse sensaciones de nistagmo y oscilaciones involuntarias de los glóbulos oculares, hay variaciones en el sueño y disminución de la capacidad de visión para los colores y las formas. (Ugarte Alba \& Mosqueda Matos; Espinoza Aguilar et al., 2018) 
Los factores de riegos físico: ruido y vibraciones constituyen riesgos importantes razón por la cual deben ser identificados, evaluados, medidos y controlados de manera efectiva que permitan asegurar el bienestar del trabajador en las labores que desempeña (Mantenimiento \& Electrógenos, 2018).

En la Tabla 1 se muestran las actividades para las que puede ser conveniente alertar sobre los efectos desfavorables de la vibración de cuerpo completo.

\begin{tabular}{|c|c|}
\hline 1 & Conducción de tractores \\
\hline 2 & $\begin{array}{l}\text { Vehículos de combate blindados (p. ej., tanques) y } \\
\text { otros similares }\end{array}$ \\
\hline 3 & $\begin{array}{l}\text { Otros vehículos todoterreno: } \\
\text { - Maquinaria de movimiento de tierras: } \\
\text { cargadoras, excavadoras, bulldozers, } \\
\text { motoniveladoras, cucharas de arrastre, } \\
\text { volquetes, rodillos compactadores } \\
\text { - Máquinas forestales } \\
\text { - Maquinaria de minas y canteras } \\
\text { - Carretillas elevadoras }\end{array}$ \\
\hline 4 & $\begin{array}{l}\text { Conducción de algunos camiones (articulados y } \\
\text { no articulados) }\end{array}$ \\
\hline 5 & Conducción de algunos autobuses y tranvías \\
\hline 6 & $\begin{array}{l}\text { Vuelo en algunos helicópteros y aeronaves de alas } \\
\text { rígidas }\end{array}$ \\
\hline 7 & $\begin{array}{l}\text { Algunos trabajadores que utilizan maquinaria de } \\
\text { fabricación de hormigón }\end{array}$ \\
\hline 8 & Algunos conductores ferroviarios \\
\hline 9 & Uso de algunas embarcaciones de alta velocidad \\
\hline 10 & Conducción de algunos ciclomotores \\
\hline 11 & Conducción de algunos turismos y furgonetas \\
\hline 12 & Algunas actividades deportivas \\
\hline 13 & Algunos otros tipos de maquinaria industrial \\
\hline
\end{tabular}

Tabla 1 Actividades para las que puede ser conveniente alertar sobre los efectos desfavorables de la vibración de cuerpo completo

Fuente)(Griffin \& Griffin, n.d.) los siguientes:

Los principales tipos de vibraciones son

- Las vibraciones del cuerpo completo ocurren cuando el cuerpo está apoyado en una superficie vibrante (por ejemplo, cuando se está sentado en un asiento que vibra, de pie sobre un suelo vibrante o recostado sobre una superficie vibrante). Las vibraciones de cuerpo completo se presentan en todas las formas de transporte y cuando se trabaja cerca de maquinaria industrial. Para el caso particular de esta investigación nos concentramos en las personas que trabajan en industrias establecidas de la región de Tecamachalco, Puebla.
- Las vibraciones transmitidas a las manos son las vibraciones que entran en el cuerpo a través de las manos. Están causadas por distintos procesos de la industria, la agricultura, la minería y la construcción, en los que se agarran o empujan herramientas o piezas vibrantes con las manos o los dedos. La exposición a las vibraciones transmitidas a las manos puede provocar diversos trastornos, a saber:

- $\quad$ Trastornos vasculares;

- $\quad$ trastornos neurológicos periféricos;

- $\quad$ trastornos de los huesos y articulaciones;

- $\quad$ trastornos musculares,

- $\quad$ otros trastornos (todo el cuerpo, sistema nervioso central).

Actividades tales como la conducción de motocicletas o el uso de herramientas vibrantes domésticas pueden exponer las manos esporádicamente a vibraciones de gran amplitud, pero solo las largas exposiciones diarias pueden provocar problemas de salud (Griffin 1990).

Dependiendo de la intensidad y duración de las vibraciones de todo el cuerpo, son también los efectos, que son usualmente trastornos de bienestar, percepción del dolor, reacción fisiológica, y la disminución en el rendimiento (Parra Parra, 2019)

Una exposición prolongada a las vibraciones está fuertemente asociada con las lumbalgias, que es una enfermedad de la columna que causa dolores en la columna baja y ocupa el tercer lugar entre las 10 principales causas de demanda de consulta en el IMSS (Instituto Mexicano del Seguro Social, 2017). A corto y mediano plazo también se pueden presentar alteraciones en las necesidades fisiológicas, alteraciones neuromusculares, alteraciones cardiovasculares, respiratorias, endócrinas y metabólicas, alteraciones sensoriales y del sistema nervioso central.

De acuerdo a la Norma Oficial Mexicana NOM-024-STPS-2001, Vibraciones Condiciones de seguridad e higiene en los centros de trabajo, los límites máximos permisibles de exposición a vibraciones en cuerpo entero se establecen en la tabla 2. 


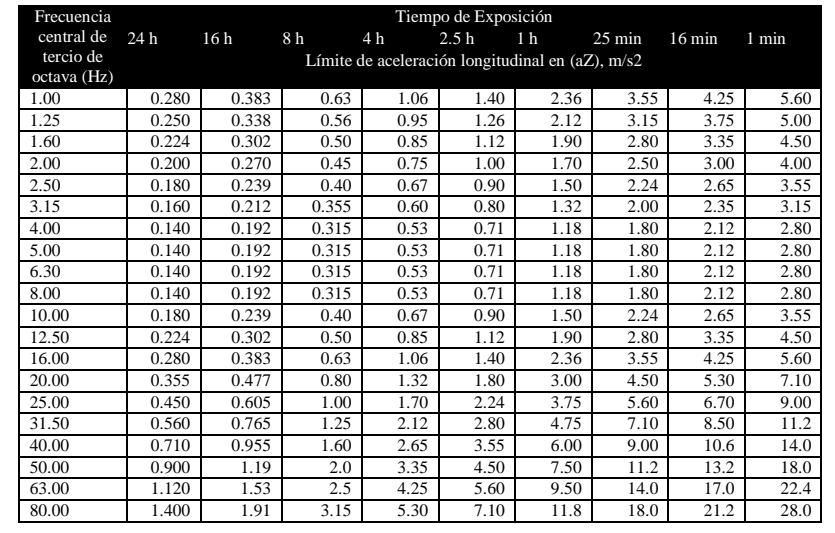

Tabla 2 Límites de aceleración longitudinal como función de la frecuencia y del tiempo de exposición (Trabajo et al., 2002)

\section{Problemática}

Los niveles de vibración en algunos centros de trabajo de la zona de Tecamachalco pueden deteriorar la salud de los trabajadores. Generalmente no existe una medición y monitoreo preciso de los niveles de vibraciones a los cuales está expuesto el trabajador, lo cual puede resultar en daños o trastornos músculoesqueléticos a largo plazo.

Los centros de trabajo de la región en general, no cuentan con ningún programa regulatorio de vibraciones y en muchos casos no tienen conocimiento de los daños que puede tener el trabajador, por ese motivo es imprescindible monitorear la aplicación de la normatividad vigente en materia de vibraciones, para evitar el incumplimiento legar y lo más importante, asegurar el bienestar de los trabajadores.

\section{Justificación}

El desarrollo de esta investigación es importante porque pretende delimitar los límites máximos de exposición de los trabajadores a las vibraciones, en magnitud y en tiempo, a fin de asegurar el bienestar de los mismos y garantizar su desempeño laboral. Por medio de la universidad se pretende realizar una concientización e implementación de este programa en las empresas de la región de Tecamachalco.

\section{Desarrollo de la investigación}

Los pasos para el desarrollo de la investigación son:

1. Investigación documental sobre las micro, pequeñas, medianas y grandes empresas de la región de Tecamachalco, para determinar las condiciones de operación de las mismas.

2. Realizar un estudio para determinar las primeras empresas que se visitarían, de acuerdo a su tamaño, localización, índice de criticidad de vibraciones, disponibilidad de tiempo y recursos.

3. Certificación de un docente en análisis de vibraciones nivel $\mathrm{I}$, ante el Vibration Institute con norma ANSI (American National Standards Institute) ISO/IEC 17024, ISO 18436-1 e ISO 18436-2. El docente Manuel Pérez Villegas obtuvo el Certificado número 2018-12615, con vigencia hasta febrero 2021, expedido por el Instituto Mexicano de Mantenimiento Predictivo, en la ciudad de Querétaro, Qro.

4. Analizar la información para determinar posibles mejoras en los procesos, viabilidad de automatización, análisis de vibraciones de los equipos y verificar que se cumpla con la norma ISO 2631-1 y con la Norma Oficial Mexicana NOM024-STPS-2001, Vibraciones Condiciones de seguridad e higiene en los centros de trabajo.

5. Visitar a las empresas para exponer los resultados del análisis de vibraciones a los encargados, con las propuestas más adecuadas para disminuir los riesgos de deterioro de la salud de los trabajadores, haciendo énfasis en los equipos o actividades de mayor riesgo y diseñar, en conjunto con la empresa, una reprogramación de actividades $\mathrm{o}$ adecuación de las áreas de trabajo que puedan ocasionar riesgos al trabajador.

6. Concientizar a los jefes o encargados, de la importancia que debe tener el cuidado del bienestar de sus empleados, obteniendo como resultado un bienestar de los mismos y en consecuencia una mayor productividad para la empresa, además de evitar consecuencias legales para la empresa por el incumplimiento de las normas o por algún accidente. 
7. Realizar un programa de seguimiento para monitorear los niveles de vibración a los cuales están expuestos los trabajadores. El seguimiento puede ser semanal, quincenal o mensual, de acuerdo a los niveles de criticidad encontrados.

8. Entrega de informes y resultados.

\section{Empresas de la región de Tecamachalco}

En la región de Tecamachalco del estado de Puebla, existen muchas empresas de todos los giros y tamaños: micro, pequeñas, medianas y grandes. En la tabla 3 se muestra la estratificación de las empresas por tamaño en México, de acuerdo a la Secretaría de Economía.

Para hacer esta catalogación se usan características cuantificables como el número de empresas ocupadas, las ventas, el valor de las inversiones o el valor de mercado de la empresa. Sin embargo, para que se pueda clasificar a todas las empresas es importante que los datos considerados sean fáciles de obtener y conocer por cualquiera (incluyendo las empresas que tienen pocos controles). Por esta razón, en muchos países se utiliza sólo el número de empleados y las ventas, ya que son datos fáciles de obtener casi a simple vista (Saavedra y Hernandez, 2008; Rafael Posada Velásquez, Óscar Cuauhtémoc Aguilar Rascón, 2016).

\begin{tabular}{|l|l|l|l|}
\hline Tamaño & \multicolumn{1}{c}{ Sector } & \multicolumn{1}{c}{$\begin{array}{c}\text { Rango de número de } \\
\text { trabajadores }\end{array}$} & \multicolumn{1}{c|}{$\begin{array}{c}\text { Rango de ventas } \\
\text { anuales }(\mathrm{mdp})\end{array}$} \\
\hline Micro & Todas & Hasta 10 & Hasta $\$ 4$ \\
\hline Pequeña & Comercio & Desde 11 hasta 30 & De $\$ 4.01$ hasta $\$ 100$ \\
\cline { 2 - 4 } & $\begin{array}{l}\text { Industria } \\
\text { servicios }\end{array}$ & Desde 11 hasta 50 & De $\$ 4.01$ hasta $\$ 100$ \\
\hline Mediana & Comercio & Desde 31 hasta 100 & De $\$ 100.01$ hasta $\$ 250$ \\
\cline { 2 - 4 } & Servicios & Desde 51 hasta 100 & \\
\cline { 2 - 3 } & Industria & Desde 51 hasta 250 & De $\$ 100.01$ hasta $\$ 250$ \\
\hline
\end{tabular}

Tabla 3 Estratificación de las empresas por tamaño en México

Fuente: Secretaria de Economía (2009)

El sector de interés para nuestra investigación corresponde a la industria metalmecánica, de manufactura y la industria de alimentos y bebidas. En general analizamos los centros de trabajo que tienen equipos que generan vibraciones, o que, por sus características de operación, pueden transmitir una cantidad importante de vibraciones a los trabajadores, ya sea por las manos o por cuerpo completo. El objetivo es determinar el nivel de vibraciones actual, compararlo con las normas vigentes y verificar si son peligrosas o no para los trabajadores.
En caso de ser potencialmente peligrosas, se darán las recomendaciones necesarias a la empresa.

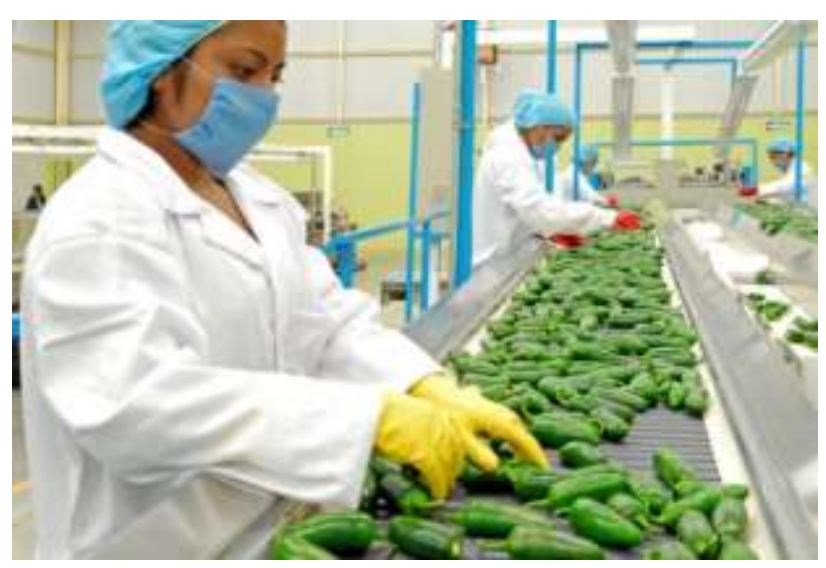

Figura 1 Vibraciones transmitidas a las manos Fuente: PRODUCMEC Exporter of Mexican Fresh Producer

Las primeras visitas se planearon para las empresas: Bachoco, Planta San Gabriel Tetzoyocan, Tecamachalco, Avitec en la ciudad de Tecamachalco y Producmec, en San Salvador Huixcolotla. Lamentablemente por la pandemia sólo se realizó la visita a Producmec. En la visita a la empresa se encontró que no existía ningún tipo de control sobre las vibraciones de los equipos ni de las transmitidas a los trabajadores.

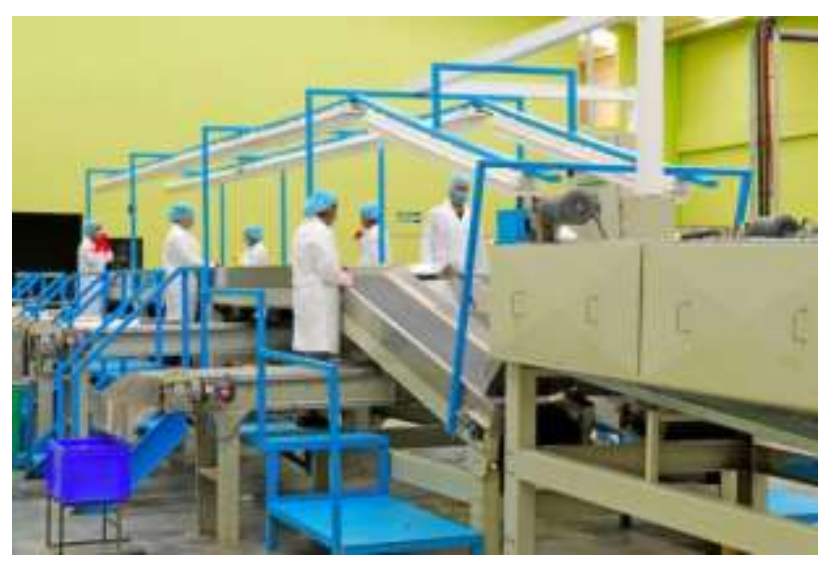

Figura 2 Vibraciones de las maquinas

Fuente: PRODUCMEC Exporter of Mexican Fresh Producer

En las figuras 1 y 2 puede observarse un contacto directo entre los rodillos con las manos de las personas, en este caso la vibración es presentada es de $0.26 \mathrm{~m} / \mathrm{s}^{2}$ en una frecuencia central de tercio de octavas de $8 \mathrm{~Hz}$. De acuerno a la NOM-024-STPS-2001, el periodo de trabajo debería de ser de 8 a 16 horas máximo, sin embargo, las jornadas de trabajo regularmente son de más de 8 horas diarias, porque la empresa trabaja de acuerdo a los pedidos recibidos diariamente.

PÉREZ-VILLEGAS, Manuel, TÉLLEZ-HERNÁNDEZ, Rubén, SÁNCHEZ-BAÑUELOS, José Sebastián y TENORIO-CRUZ, Fermín. Deterioro de la salud por causa de las vibraciones mecánicas en los centros de trabajo de la zona de Tecamachalco. Revista de Ciencias de la Salud. 2020 
Como medida de prevención, la empresa tiene una rotación de personal, para que las personas no estén siempre en contacto con la máquina, pero hay ocasiones en que deben estar hasta 12 horas o más trabajando en la misma máquina.

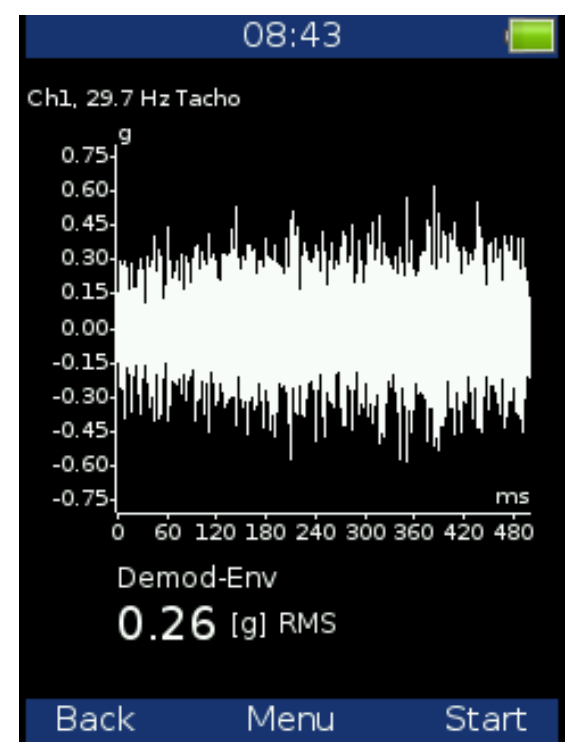

Figura 3 Lectura de vibración de $0.26[\mathrm{~g}] \mathrm{RMS}\left(\mathrm{m} / \mathrm{s}^{2}\right)$ Fuente: Analizador de vibraciones ADASH 4300 VA3

Se le recomendó a la empresa asegurarse de que la exposición a las vibraciones diarias no excediera de 8 horas diarias. Se hizo énfasis en los beneficios de la implementación de nuestro programa de monitoreo de vibraciones en la empresa, particularmente en el bienestar de los trabajadores y en alargar la vida útil de los equipos. A raíz de eso, se puro formalizar un convenio de colaboración con la empresa. El equipo con el cual se realizan los estudios de vibraciones es el analizador de vibraciones para diagnóstico de maquinaria ADASH 4300 VA3, con acelerómetro $100 \mathrm{mV} / \mathrm{g}$, que es un potente analizador de vibraciones multicanal de uso industrial.

\section{Resultados}

Los resultados aún son parciales, porque debido a la pandemia de COVID-19 no se pudo terminar la investigación como se tenía planeada, pero de acuerdo a los primeros resultados ya obtenidos de parte de la empresa Producmec, el objetivo de la investigación se cumplió satisfactoriamente, porque se reunieron los elementos suficientes para implementar un programa de monitoreo y control de vibraciones en esta empresa de la región de Tecamachalco, además de la firma de un convenio de colaboración que beneficia ambas partes.

\section{Conclusiones}

Concluimos que esta investigación es viable y los primeros resultados obtenidos son satisfactorios de acuerdo al objetivo principal:

- $\quad$ Se identificaron las principales fuentes de vibraciones, su magnitud y riesgo para los trabajadores.

- Se realizó un programa de seguimiento para monitorear los niveles de vibración a los cuales están expuestos los trabajadores.

- Mediante las evaluaciones de vibraciones de cuerpo completo y de manos, realizadas en los trabajadores que tienen contacto con los equipos, se identificaron los niveles de vibración.

- Dado que la exposición continua a las vibraciones aumenta el riesgo por vibración, los horarios de trabajo se modificaron en beneficio de la salud del trabajador.

- Se firmó un convenio de colaboración para brindar el asesoramiento adecuado a la empresa y capacitar en métodos de trabajo correctos y seguros.

\section{Referencias}

Castro, A., Jesús, G. De, Oropesa, M., \& Reyes, A. (2016). Evaluación de las vibraciones globales transmitidas a trabajadores en una empresa agroindustrial productora de azúcar. Salud de Los Trabajadores, 24(1), 27-37.

Espinoza Aguilar, Y., Luna Florin, A., Buele Calderón, M., \& Merchan Ochoa, A. (2018). Diagnóstico de las afectaciones socioambientales por el ruido en el procesamiento de cacao de la corporación CAORO en el sitio Río Negro. Revista San Gregorio, 1(22), 22-29. https://doi.org/10.36097/rsan.v1i22.596

Griffin, M. J., \& Griffin, M. J. (n.d.). Director del capítulo.

Jesús, A. G. De, \& Ciro, M. (2016). Evaluación de la exposición al riesgo por vibraciones en el segmento mano brazo en compañías del sector metalmecánico. Medicina y Seguridad Del Trabajo, 62(245), 327-336.

Mantenimiento, O. Y., \& Electrógenos, D. E. G. (2018). ELECTRÓNICA E INDUSTRIAL. 
Parra Parra, J. A. (2019). Evaluación de vibraciones de cuerpo completo en los trabajadores que manipulan el equipo caminero del GAD municipal de Latacunga. 2. http://repositorio.unan.edu.ni/2986/1/5624.pdf

Rafael Posada Velásquez, Óscar Cuauhtémoc Aguilar Rascón, N. B. P. A. (2016). Análisis sitemático de la micro y pequña empresa en México (Issue December).

Trabajo, D., Seguridad, R. F. De, Mexicana, N. O., Administrativo, P., Regulatoria, F. D. M., Higiene, V. D. E. S. E., \& Centros, E. N. L. O. S. (2002). Secretaria del trabajo y prevision social. 